\title{
Restoration Aid Expert System for Power Systems
}

\author{
Heung-Jae Lee ${ }^{l}$ and Jung-Hyun $\mathrm{Oh}^{2, *}$ \\ ${ }^{1}$ Department of Electrical Engineering, Kwangwoon University, 01897 Seoul, Korea \\ ${ }^{2}$ School of Robotics, Kwangwoon University, 01897 Seoul Korea
}

\begin{abstract}
This paper propose an intelligent restoration aid system that assists the decision of power system operators for reliable and fast restoration processes when a blackout happened in massive power systems. This system consists of a topology processor and intelligent restoration aid system. The topology processor identifies the real-time embedded topology structure between power system facilities and determines the power outage section. The power system restoration aid system determines the feasible restoration path in terms of overloads and real power flow. Case studies demonstrate a promising possibility of practical application.
\end{abstract}

\section{Introduction}

Recently, unexpected power system blackouts have frequently occurred in many counties, leading to great socio-economic losses. When a blackout happens in the bulk power system, it is very important to provide fast and reliable restorative operation while preventing cascading blackouts in power system.

The self-healing is a high-end technology that accurately recognizes power network disturbances and maintains the optimal state. Also, it is the final goal of the smart grid project which is currently in the progress of being developed worldwide. In order to have the ability of self- healing, numerous precision sensors, advanced operations in each hierarchy, and automatic control systems are ultimately required.

However, so far the performance of hierarchical cooperative operation technology should be improved. Especially the power system restoration has entirely relied on the operator's heuristic knowledge and judgment. Because of non-linear characteristics of power system, it is extremely difficult to set out reliable restoration plans.

To prevent the re-trip of circuit breakers caused by overvoltages or overloads during restoration processes, analytical verifications are often required since it may enormously delay restoration time. Therefore, restoration guidance systems that may uphold power system operator's judgment during restoration processes are necessary in the long run. Some countries had already developed the restoration guidance systems and applied them to the transmission systems, or substationdistribution systems. In Korea, basic researches for distribution systems and $154 \mathrm{kV}$ substations had been performed but few basic researches in the level of transmission system had been executed so far. In recent years, necessity of restoration guidance systems for power system has been invoked since several blackout incidents broke out in Korean power system.
In this paper, the power system restoration guidance systems are presented. This system consists of three major subsystems such as a topology processor, main restoration guidance expert system, and voltage control expert system. The topology processor identifies the realtime embedded topology structure between power system facilities and at the same time determines the power outage section. The voltage control expert system, which is not introduced here, adjusts abnormal voltages that may happen during restoration processes, which is especially developed based on the voltage sensitivity analysis. The power system restoration guidance expert systems establish the fast and feasible restoration path by using DC power flow. The Human Machine Interface (HMI) is built by combining three major subsystems with simulator for various case studies. Regarding the HMI, special dynamic coloring function is also developed using AI technique. The setting of developed dynamic coloring can be divided by static attribution settings for representing constant values and dynamic attribution settings for representing continuously changed values [1]. Attribution of facilities is obviously represented by setting of tag values, type and width of lines, characters and circles. Dynamic attribution is represented by the flicker or change of colors according to the pre-defined rules. The power system restoration simulator using dynamic coloring is vividly demonstrated as shown in the case study. Here, the result of case study will demonstrate a potential extension of practical application.

\section{Topology Processor}

A power system is generally made up of generation, transmission and substation facilities. It is very vital to identify the topology structure between transmission and substation facilities when power system disturbances are created by malfunctions or non-operations of power system devices.

* Corresponding author: ihyunoh@kw.ac.kr 
Topology structure identification of transmission lines and substations is regarded as a step of implementing mechanisms, where power system operators intuitively detect each component's connection relationship represented in a single-line diagram, via a computer program. Double-bus $1.5 \mathrm{CB}$ structure of $345 \mathrm{kV}$ substation is shown in Fig. 1.

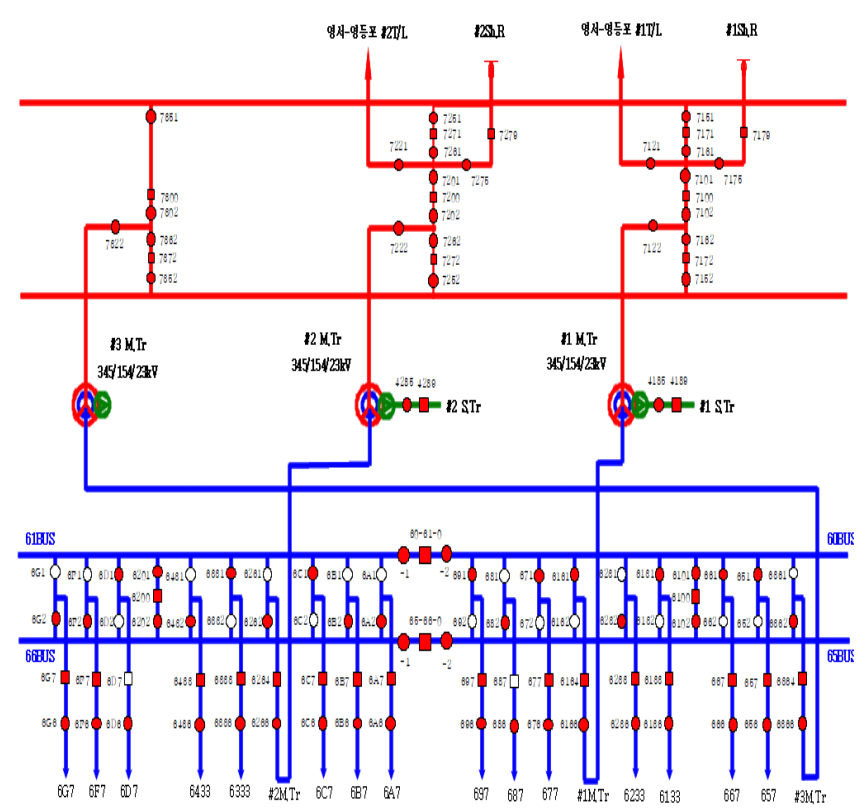

Fig. 1. Structure of a $345 \mathrm{kV}$ substation.

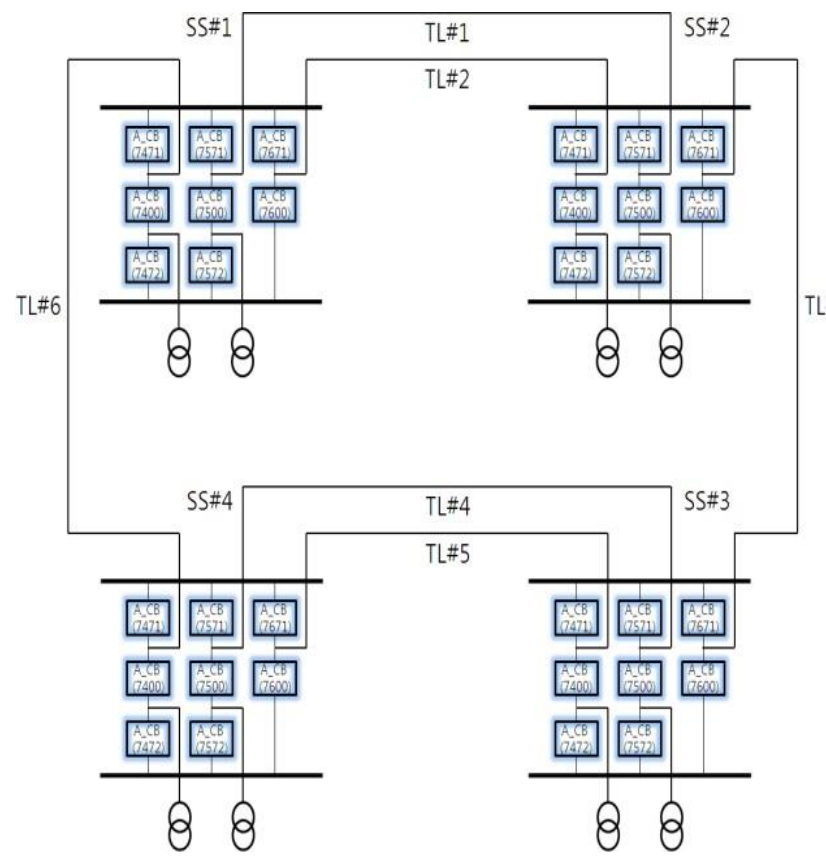

Fig. 2. Aggregated transmission system.

Under such a complicated switching structure condition, the topology processor enables computers to identify the transmission network connections [2]. Data structures are effectively defined as aggregated $\mathrm{CB}$ to find topology structure of transmission network [3].
Furthermore, the dual searching strategy is adopted to represent the topology structure of transmission facilities [4]. The topology structure of aggregated transmission system is schematically displayed in Fig. 2.

\section{Restoration Aid Expert System}

The main restoration aid expert system is the core expert system that determines the restoration path when a blackout occurred in power system [5]. The structure of this system is a rule-based expert system as seen in Fig. 3. The knowledge base comprises the rule base that stores if-then type of knowledge and the database that retains static data and dynamic data as well. The inference engine is a process that solves given problems and derives a solution from the rules of knowledge base and the facts accumulated in the database.

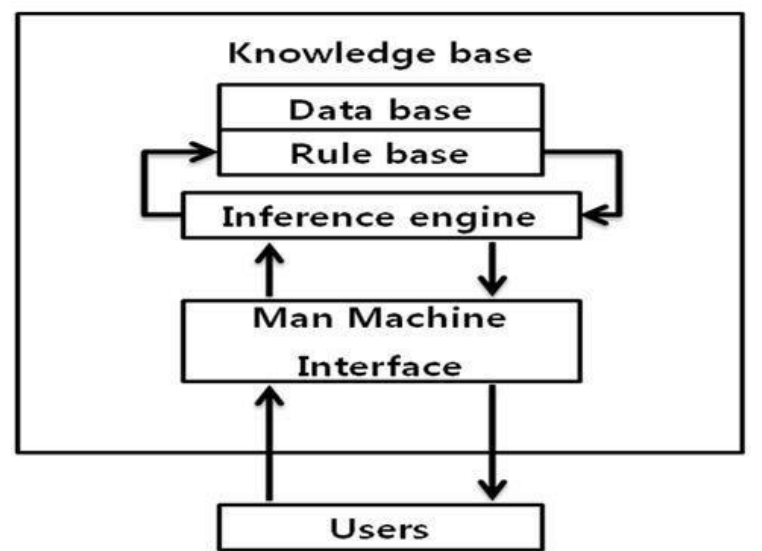

Fig. 3. Structure of rule based expert system.

In the expert system, the problem representation method is simply classified into the state space representation and the problem reduction technique. In general, the problem reduction technique has been frequently used in power system. Nevertheless, the power system restoration guidance system was developed by making use of the state space model due to the nature of problem. State space of power system restoration problem is briefly expressed in Fig. 4. For the efficient search, the best-first search method was chosen to search an optimal combination of switching.

As to the rule base, five groups of rule set are developed as follows.

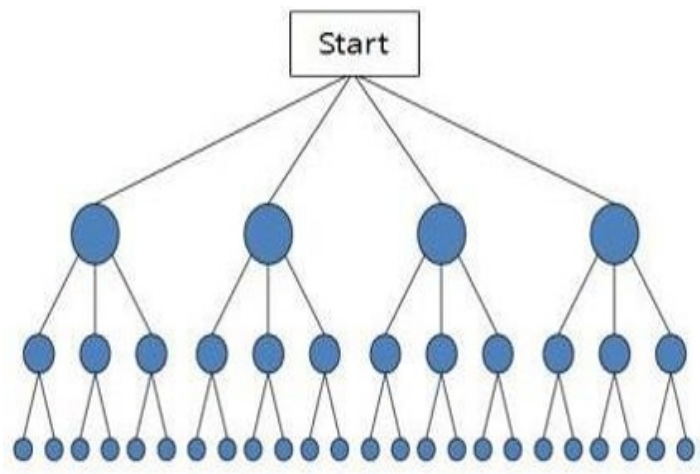

Fig. 4. State space of restoration problem in power system 
1) Rules for recognizing blackout section

Rules for restoration from massive blackout 12 linguistic rules are developed and an example is as following.

- If there is a black-start generator in the blackout section, then turn on the generator at first.

2) Rules for power supply to load from non-blackout region. 6 rules are developed and an example is as following. If there exist one or more restorable path, then select a restorable path that power supply ratio is greater than other restorable paths.

3) Rules for partitioning load in blackout region. 3 rules are developed to prevent overloads.

4) Rules for increasing power capacity to supply blackout region.

8 rules are developed and an example is as following.

If there exist two or more adjustable generators, then select the larger capacity generator

\section{Case Study}

To verify the performance of the suggested power system restoration guidance systems, serious power outage of Dongseoul, Korea in 1994 is assumed. The massive blackout is attributable to the trip of $154 \mathrm{kV}$ transmission line and the developed topology processor recognizes the power outage section as shown in Fig. 5. If a user selects the restoration menu in the top menu of HMI, restoration guidance systems suggests detailed restoration path, which are displayed in the pop-up window, based on rules of knowledge base in the expert system and the calculation of power flows as shown in Fig. 6. The detailed restoration sequence in the pop-up window in fig. 6 , which are written by Korean, is shown in the Table 1 .

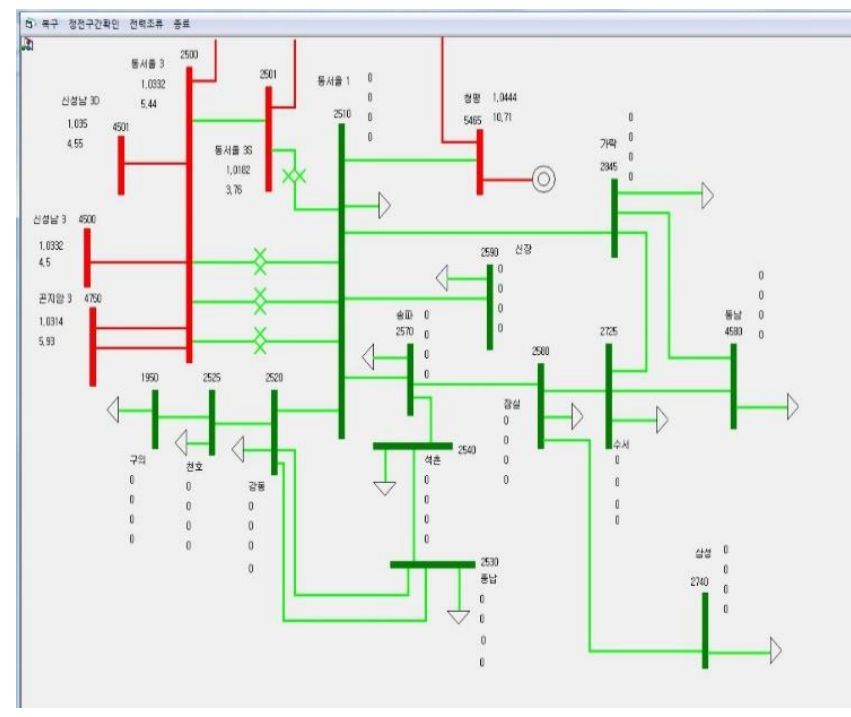

Fig. 5. Display of blackout case study in Dongseoul
Table 1. The restoration path provided by system

\begin{tabular}{|c|l|}
\hline STEP & RESTORATION PATH \\
\hline STEP 1 & $\begin{array}{l}\text { T/L Energizing : Dongseoul1-Cheongpyung PP } \\
\text { Load pick-up : Dongseoul1 }\end{array}$ \\
\hline STEP 2 & $\begin{array}{l}\text { T/L Energizing : Dongseoul1-Garak } \\
\text { Load pick-up : Garak }\end{array}$ \\
\hline STEP 3 & $\begin{array}{l}\text { T/L Energizing : Garak-Suseo } \\
\text { Load pick-up : Suseo }\end{array}$ \\
\hline STEP 4 & $\begin{array}{l}\text { T/L Energizing : Suseo-Dongnam } \\
\text { Load pick-up : Dongnam }\end{array}$ \\
\hline STEP 5 & T/L Energizing : Garak-Dongnam \\
\hline STEP 6 & $\begin{array}{l}\text { T/L Energizing : Dongseoul3S-Dongseoul TR } \\
\text { T/L Energizing : Dongseoul1-Dongseoul3 }\end{array}$ \\
\hline STEP 7 & $\begin{array}{l}\text { T/L Energizing : Dongseoul1-Sinjang } \\
\text { Load pick-up : Sinjang }\end{array}$ \\
\hline STEP 8 & $\begin{array}{l}\text { T/L Energizing : Dongseoul1-Songpa } \\
\text { Load pick-up : Songpa }\end{array}$ \\
\hline STEP 9 & $\begin{array}{l}\text { T/L Energizing : Songpa-Jamsil } \\
\text { Load pick-up : Jamsil }\end{array}$ \\
\hline STEP 10 & $\begin{array}{l}\text { T/L Energizing : Jamsil-Suseo, Jamsil-Samsung } \\
\text { Load pick-up : Samsung }\end{array}$ \\
\hline STEP 11 & $\begin{array}{l}\text { T/L Energizing : Dongseoul1-Gangdong } \\
\text { Load pick-up : Gangdong }\end{array}$ \\
\hline STEP 12 & $\begin{array}{l}\text { T/L Energizing : Songpa-Seokchon } \\
\text { Load pick-up : Seokchon }\end{array}$ \\
\hline STEP 13 & $\begin{array}{l}\text { T/L Energizing : Gangdong-Cheonho } \\
\text { Load pick-up : Cheonho }\end{array}$ \\
\hline STEP 14 & $\begin{array}{l}\text { T/L Energizing : Cheonho-Guui } \\
\text { Load pick-up : Guui }\end{array}$ \\
\hline STEP 15 & $\begin{array}{l}\text { T/L Energizing : Seokchon-Pungnab, Pungnab-Gangdong } \\
\text { Load pick-up : Pungnab }\end{array}$ \\
\hline
\end{tabular}

Finally, the restoration is completed along with a restoration path in Fig. 7.

If a user selects another restoration path different from the path provided by the restoration guidance systems, overvoltages or overloads may happen. Detailed examples are not shown due to page limits. From this perspective, it is apparently pointed out that the power system restoration aid systems is useful to generate a correct restoration path.

\section{Conclusion}

In this paper, the power system restoration guidance systems have been addressed for power system operator's reliable and fast restoration in case of the power system outages.

As mentioned earlier, the topology processor accurately recognized the power system outage section. The power system restoration guidance expert systems are integrated with the voltage control expert system and then the restoration path for preventing overvoltages and overloads is proposed for power system operators.

Besides, the foregoing analysis with the outage data from Korea has proved that the suggested restoration guidance systems might realize reliable and fast restoration operations enough to be applicable to the real power system. 


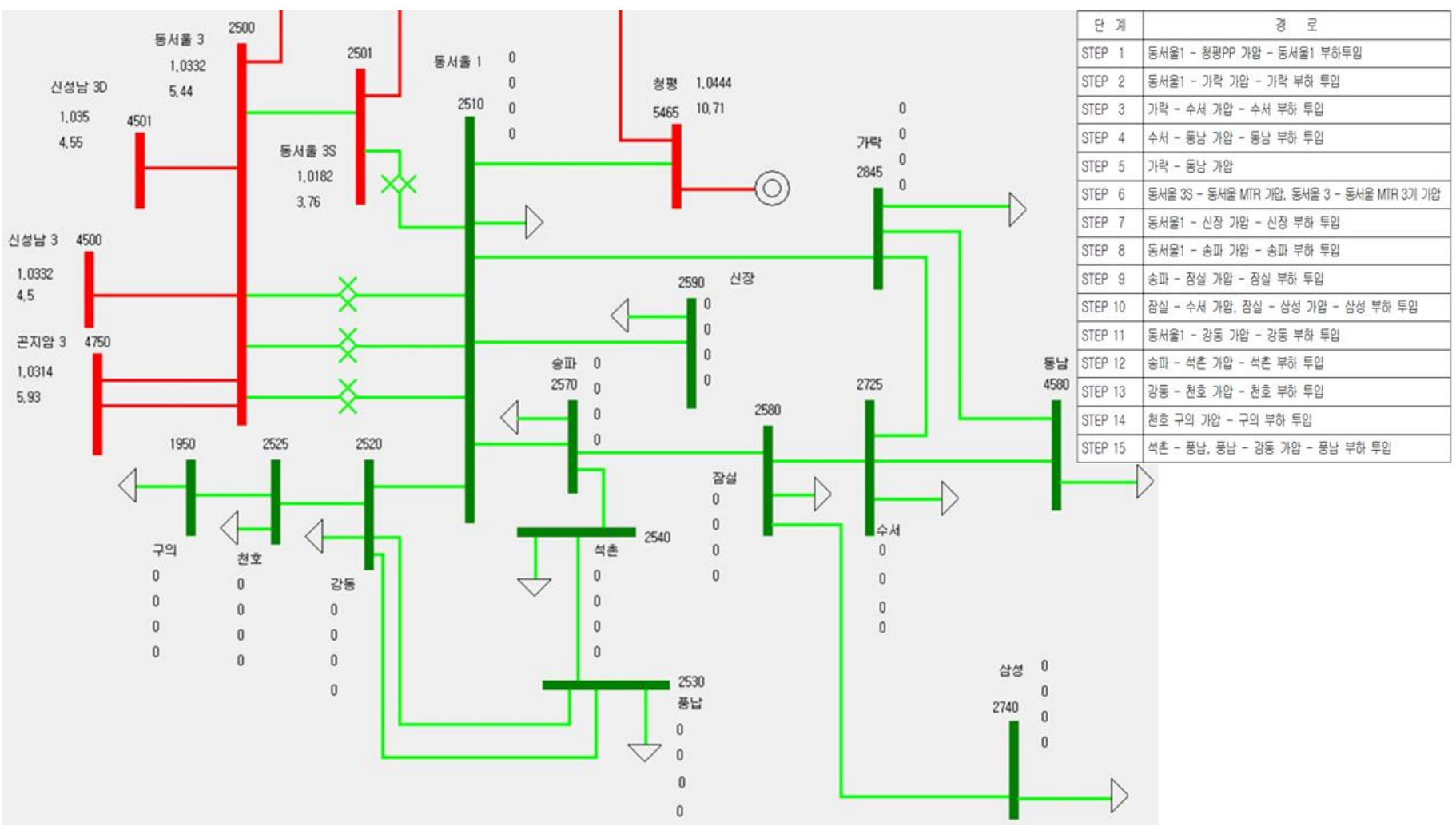

Fig. 6. Display of restoration path provided by system

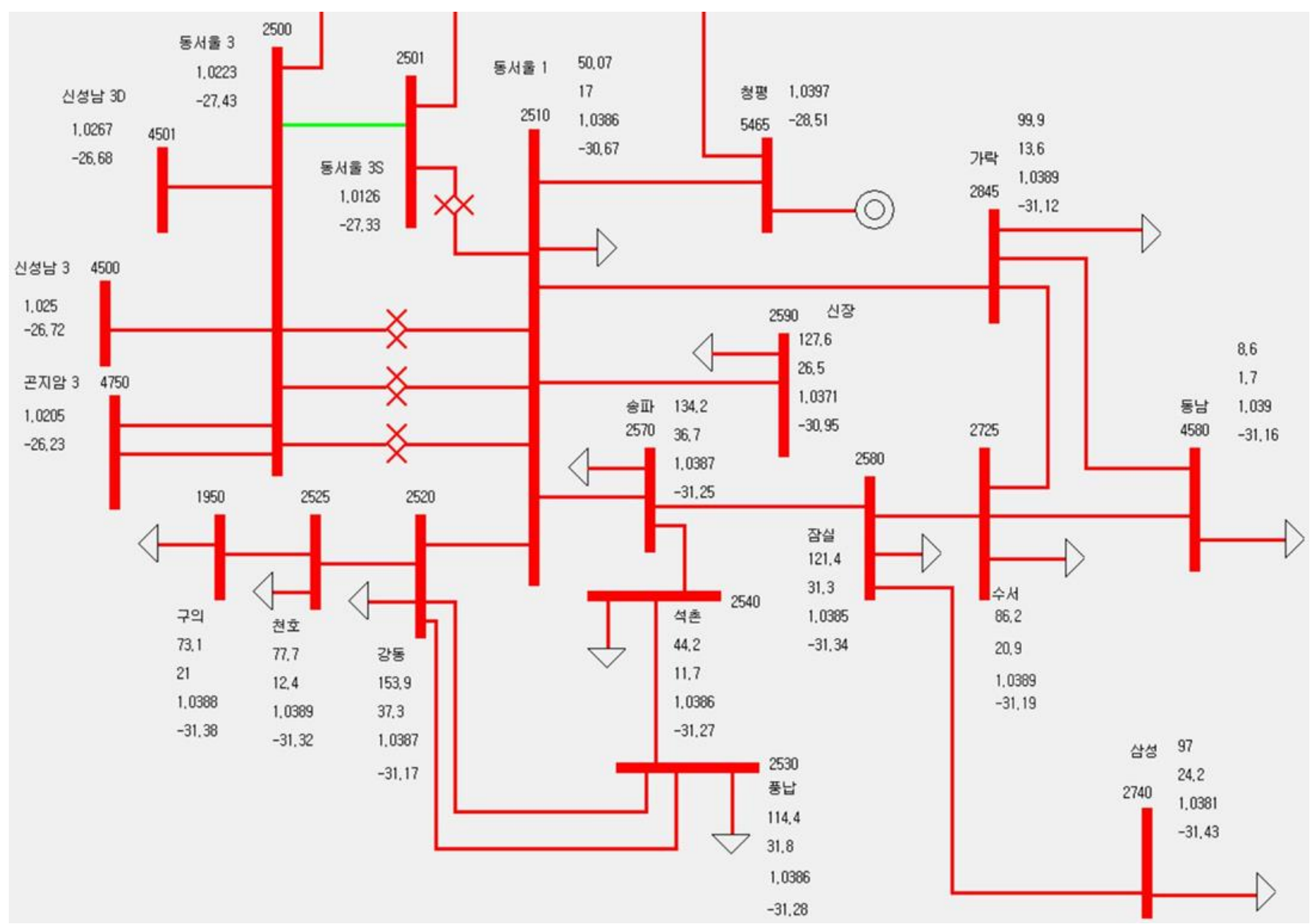

Fig. 7. Display after full restoration 
This work was supported by Korea Institute of Energy Technology Evaluation and Planning (KETEP) and the Ministry of Trade, Industry \& Energy (MOTIE) of the Republic of Korea (No. 20174010201620), and Korea Electric Power Corporation. (R17XA05-20)

\section{References}

1. Heung-Jae Lee, at. al., "Development of the Transmission System Restoration Guidance System and Simulator", the final report supported by $M K E$, p. 100-106 (2012)

2. Heung-Jae Lee, at. al., "Development of the Transmission System Restoration Guidance System and Simulator", the final report supported by MKE, $\mathrm{p}$. 81-99 (2012)

3. Chan-Ho Lim, Won-Kun Yu, Eun-Jae Lee, HeungJae Lee and Jun-Ho Park, "A Study on the Topology Processor for $765 \mathrm{kV}$ Substation in Korea," in Proceedings of International Middle East Power System Conference, Cairo University, Egypt, (2010)

4. Heung-Jae Lee, Chan-Ho Lim, Soo-Gil Lee, KyunDo Kim, In-Soo Wang, Hyun-Jae Kang and WonKun Yu, "Identification and correction of topology errors in distribution substations", ICIC Express Letters, p.1321- 1326 (2009)

5. Heung-Jae Lee, at. al., "Development of the Transmission System Restoration Guidance System and Simulator", the final report supported by MKE, $\mathrm{p}$. 28-39 (2012) 